\title{
The AKARI Far-Infrared Surveyor (FIS): all-sky Diffuse Map
}

\author{
Shinya Komugi ${ }^{1,2,3}$, Yasuo Doi ${ }^{4}$, Makoto Hattori ${ }^{5}$, \\ Yoshimi Kitamura ${ }^{1}$, Takafumi Otsubo ${ }^{5}$, Masahiro Tanaka ${ }^{6}$, \\ Norio Ikeda ${ }^{1}$, Daisuke Kato ${ }^{1}$ and Takao Nakagawa ${ }^{1}$ \\ ${ }^{1}$ Institute of Space and Astronautical Science, Japan Exploration Agency, 3-1-1 Yoshinodai, \\ Chuo-ku, Sagamihara, Kanagawa, Japan, email: skomugi@ir.isas.jaxa.jp \\ ${ }^{2}$ Joint ALMA Observatory, Alonso de Cordova 3107, Vitacura, Santiago, Chile \\ ${ }^{3}$ National Astronomical Observatory of Japan, Osawa 2-21-1, Mitaka, Tokyo, Japan \\ ${ }^{4}$ Department of Earth Science and Astronomy, Graduate School of Arts and Sciences, The \\ University of Tokyo, Komaba 3-8-1, Meguro, Tokyo 153-8902, Japan \\ ${ }^{5}$ Astronomical Institute, Graduate School of Science, Tohoku University, Aramaki, Aoba-ku, \\ Sendai, 980-8578, Japan \\ ${ }^{6}$ Center for Computational Sciences, Tsukuba University, 1-1-1 Tennodai, Tsukuba-city, \\ Ibaraki 305-8577, Japan
}

\begin{abstract}
The infrared astronomical satellite AKARI performed an all sky survey at six infrared bands. We report here on the calibration of the all-sky image data, observed in the four long wavelength bands with the FIS instrument (AKARI Far-infrared All Sky Survey : AFASS). The preliminary image attains a calibration uncertainty and sensitivity of better than $\sim 30 \%$ and $\sim 10 \mathrm{MJy} \mathrm{str}{ }^{-1}$, respectively, for all four bands. The point spread function (PSF) is obtained via a stacking technique. The data are shown to be useful for exploring the internal structure and dust spectral energy distributions (SEDs) of nearby galaxies.
\end{abstract}

Keywords. surveys, atlases, (ISM: ) dust, extinction, galaxies: ISM, techniques: image processing

\section{Introduction}

The all sky survey conducted by AKARI (Murakami et al., 2007) observed $94 \%$ of the sky during its cold phase (Nakagawa et al., 2007) in 2006 Feb. - 2007 Aug., and released its first point source catalog in 2010 (Yamamura et al., 2010). The Far Infrared Surveyor (FIS) (Kawada et al., 2007) is one of the two focal plane instruments onboard AKARI observing the 65, 90, 140 and $160 \mu \mathrm{m}$ bands. AKARI scanned the sky in a ecliptic orbit, with the telescope always pointing away from the earth. At an altitude of $\sim 700 \mathrm{~km}$, AKARI made an orbit around the earth in 100 minutes, which translates into a scan speed of $\sim 220^{\prime \prime} \mathrm{s}^{-1}$. The observing strategy is explained in Kawada et al. (2007).

\section{Data Processing}

The data were pre-processed using the AKARI pipeline tool optimised for point source extraction (Yamamura et al., 2009), which corrected for linearity and sensitivity drifts of the detectors, rejection of cosmic ray hits, and intrumental effects. Further reduction optimised for imaging large spatial scale structures was utilized. This diffuse pipeline additionally despikes data, corrects for the transient response of the far-IR photoconductor detectors, and destripes for artifacts made by sensitivity drifts along the scan. Details 

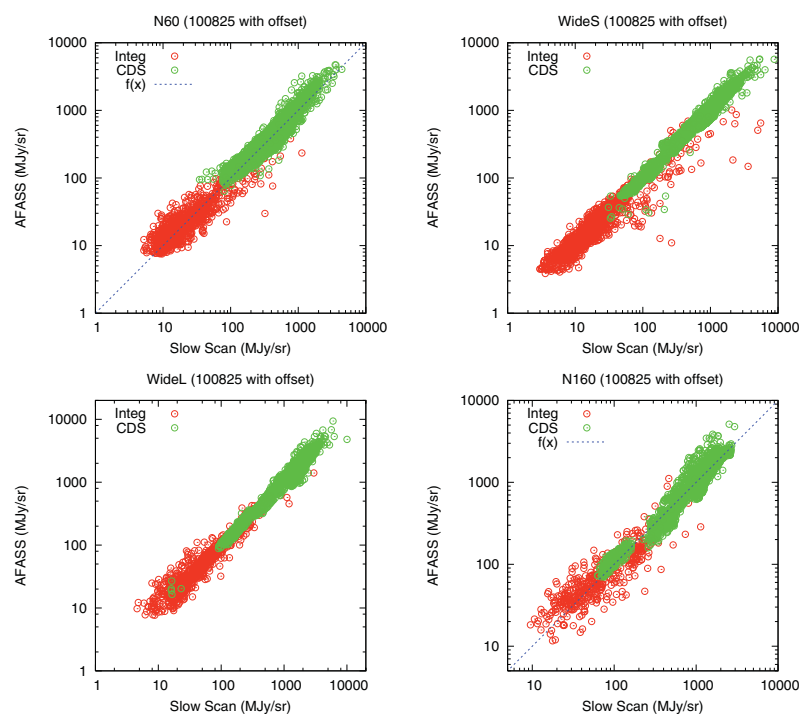

Figure 1. Comparison of flux from AKARI in the slow scan mode and all sky survey (AFASS) for the four FIS bands. Dark and light shades points correspond to nearby galaxies and Galactic regions, respectively.

of the diffuse map reduction pipeline are presented in Doi et al. (2009a) and Doi et al. (2009b).

\section{Calibration}

AKARI also conducted pointed "slow scan" observations toward selected objects. The slow-scan observations use slow scan speed $\left(8^{\prime \prime} \mathrm{s}^{-1}\right.$ or $\left.15^{\prime \prime} \mathrm{s}^{-1}\right)$, and result in a sensitivity 1-2 orders of magnitude better than the AFASS which has a far faster scan speed. Shirahata et al. (2009) calibrated slow scan images using aperture photometry on astronomical point sources, mostly consisting of stars and asteroids. Calibration of this slow scan data are found to be accurate to $14 \%, 13 \%, 10 \%$, and $50 \%$ for the $65 \mu \mathrm{m}, 90 \mu \mathrm{m}$, $140 \mu \mathrm{m}$, and $160 \mu \mathrm{m}$ bands, respectively.

The slow scan observations and the AFASS imaging share all systems except for the scanning strategy, and as such makes it the best dataset to compare with. Fifty-five nearby galaxies and 8 Galactic regions observed in the slow scan mode, were used to calibrate the all sky image against.

The AFASS images were constructed to the same grid as the slow scan images, then Zodiacal light for all of the bands were subtracted using the Kelsall (Kelsall et al., 1998) or Wright (Wright et al., 1998) models for each pixel.

The AFASS output pixel values which are in adu units, are converted to units of MJy str $^{-1}$ by deriving scaling factors from comparison of the corresponding slow scan image pixels for each band. An average value of the scaling factors is used for all of the data. Only data pixels with values over $3 \sigma$ of the sky level are used here.

Figure 1 shows the comparison of pixel-based values between slow scan and calibrated AFASS data, and their $1 \sigma$ dispersion in Table 1 . The data are well correlated with a slope of 1 , extending down to $\sim 10 \mathrm{MJy} \mathrm{str}^{-1}$, indicating the characteristic sensivity of the dataset. 
Table 1. The FWHMs of the Point Spread Functions (PSFs), and the calibration uncertainty for the four FIS bands. The FWHMs for in-scan $\left(\mathrm{FWHM}_{i n}\right)$ and cross-scan $\left(\mathrm{FWHM}_{\text {cross }}\right)$ directions are also shown. $\mathrm{N}_{\text {source }}$ shows the number of sources used for making the PSFs.

\begin{tabular}{lcccccc}
\hline band & center wavelength & FWHM & FWHM $_{\text {in }}$ & FWHM $_{\text {cross }}$ & $\mathrm{N}_{\text {source }}$ & $1 \sigma$ error \\
\hline N60 & $65 \mu \mathrm{m}$ & $39^{\prime \prime}$ & $38^{\prime \prime}$ & $41^{\prime \prime}$ & 28 & $25 \%$ \\
WideS & $90 \mu \mathrm{m}$ & $60^{\prime \prime}$ & $58^{\prime \prime}$ & $61^{\prime \prime}$ & 104 & $23 \%$ \\
WideL & $140 \mu \mathrm{m}$ & $87^{\prime \prime}$ & $96^{\prime \prime}$ & $79^{\prime \prime}$ & 53 & $15 \%$ \\
N160 & $160 \mu \mathrm{m}$ & $78^{\prime \prime}$ & $76^{\prime \prime}$ & $80^{\prime \prime}$ & 100 & $32 \%$ \\
\hline
\end{tabular}

\section{Point Spread Function}

The Point Spread Functions (PSFs) for each band are constructed based on AFASS images of "emission line galaxies" which are indentified based on the SIMBAD astronomical database, and detected both by the "AKARI/FIS Point Source Catalogue" (Yamamura et al., 2010) and the "IRAS Point Source Catalogue" as a single point source. After the flux of each source is normalized, all images are aligned by scan-direction and spatially aligned based on the positions of the sources, and then combined by averaging to make the PSF images. The FWHMs of the PSFs and the number of sources used for making the PSFs are listed in Table 1. The FWHMs for in-scan and cross-scan direction are also tabulated.
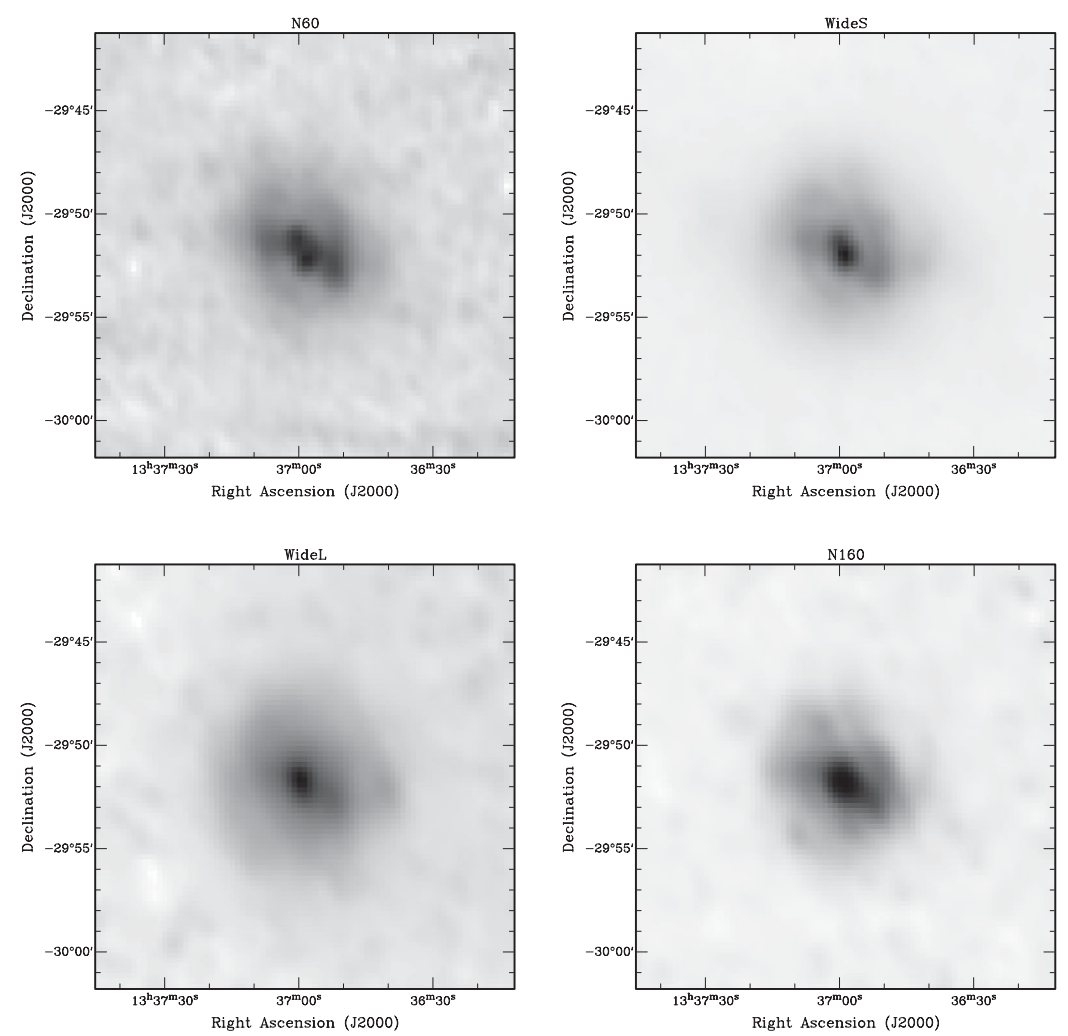

Figure 2. The galaxy M 83 in AFASS. Clockwise from top left; N60, WideS, WideL and N160 bands. 

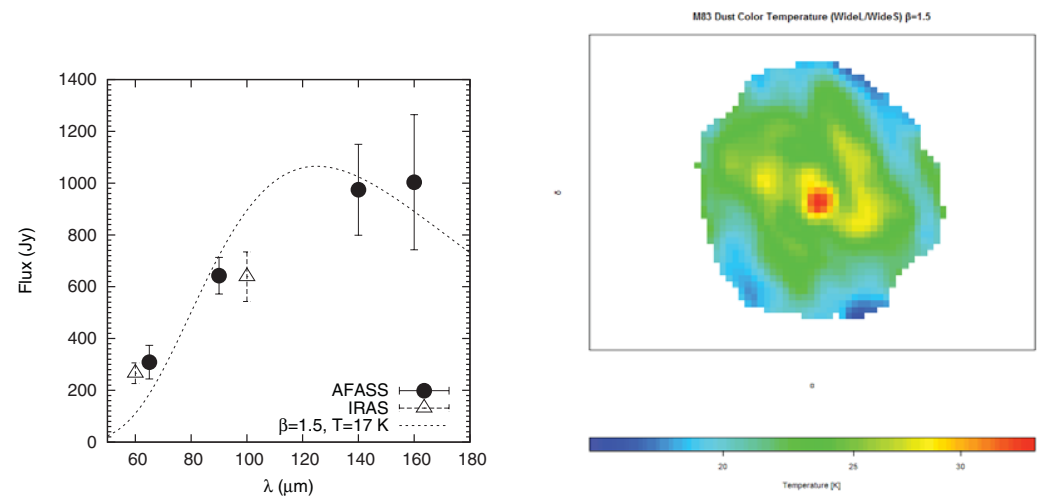

Figure 3. Left : The far-IR SED of M 83 from AFASS, with IRAS 60 and $100 \mu \mathrm{m}$ points. Right: The color temperature distribution, from 90 and $140 \mu \mathrm{m}$ assuming a dust emissivity index of 1.5 .

\section{Nearby Galaxies in the Survey}

An example of a galaxy observed in AFASS, M 83 is presented in figure 2 for the four FIS bands. The structure of the disk, the central intense star formation and the bar structure are cleary resolved. This enables a resolved study of dust in the galaxy, which was not possible with previous all sky image surveys such as IRAS.

Further, the four far-IR bands are able to characterize the spectral energy distribution (SED) up to $160 \mu \mathrm{m}$, thus probing the colder component than that observed by IRAS. Figure 3 shows the global SED of M 83, along with fluxes from IRAS at 60 and $100 \mu \mathrm{m}$. The flux are consistent at the comparable wavelengths. The longest and shortest wavelength data deviate from a best-fit modified blackbody, implying that a single dust temperature may not be sufficient for describing the full far-IR SED (c.f., Komugi et al., 2011). Nevertheless, a simple ratio between the two high sensitivity bands (WideS and WideL) can be used to give a characteristic dust temperature distribution (as have been done extensively in the literature) within the galaxy, as shown in the right panel of figure 3. The spiral structure is clearly seen as a higher temperature component.

\section{References}

Doi, Y., et al. 2009, The Next-Generation Infrared Space Mission: SPICA, 4018

Doi, Y., et al. 2009, Astronomical Society of the Pacific Conference Series, 418, 387

Doi, Y., et al. 2009, in: Proc. Workshop The Next-Generation Infrared Space Mission: SPICA, 4018

Kaneda, H., Onaka, T., Suzuki, T., Takahashi, H., \& Yamagishi, M. 2009, Astronomical Society of the Pacific Conference Series, 418, 197

Kawada, M., et al. 2007, PASJ, 59, 389

Kelsall, T., et al. 1998, ApJ, 508, 44

Komugi, S., et al. 2011, this volume

Murakami, H., et al. 2007, PASJ, 59, 369

Nakagawa, T., et al. 2007, PASJ, 59, 377

Shirahata, M., et al. 2009, PASJ, 61, 737

Verdugo, E., Yamamura, I., \& Pearson, C. P. 2007, AKARI FIS Data Users Manual, Version 1.3, http://www.ir.isas.jaxa.jp/ASTRO-F/Observation

Wright, E. L. 1998, ApJ, 496, 1

Yamamura, I., et al. 2009, Astronomical Society of the Pacific Conference Series, 418, 3

Yamamura, I., Makiuti, S., Ikeda, N., Fukuda, Y., Oyabu, S., Kota, T., \& White, G. J., 2010 